\title{
Pullback attractors for a two-phase flow model in an infinite delay case
}

Min Yang*(1)

\section{*Correspondence:}

minyang@hhu.edu.cn

College of Aerospace Engineering,

Nanjing University Of Aeronautics

and Astronautics, Nanjing, 210016,

China

Department of Mathematics,

College of Sciences, Hohai

University, Nanjing, 210098, China

\begin{abstract}
In this paper we study the existence of solutions for a coupled

Allen-Cahn-Navier-Stokes model in two dimensions with an external force containing infinite delay effects in the weighted space $C_{\delta}(\mathbb{Y})$. We prove the existence of pullback attractors for the dynamical system associated to the problem under more general assumptions.
\end{abstract}

Keywords: Allen-Cahn-Navier-Stokes; infinite delays; pullback attractors

\section{Introduction}

Diffuse interface models are well-known tools to describe dynamics of complex fluids ([1, 2]). For instance, this approach is used in [3] to describe cavitation phenomena in a flowing liquid. The resulting model essentially consists of the Navier-Stokes equations suitably coupled with the well-known phase-field system. In the isothermal compressible case, existence of a global weak solution for such a system has been recently proved in [4]. In the incompressible isothermal case, neglecting chemical reactions and other forces, the model reduces to an evolution system which governs the fluid velocity $\boldsymbol{u}$ and the order parameter $\phi$. This system can be written as a Navier-Stokes equation coupled with a convective Allen-Cahn equation.

In [5, 6], Gal and Grasselli proved that the initial and boundary value problem generates a strongly continuous semigroup on a suitable phase space which possesses the global attractor $A$ and establish the existence of an exponential attractor $E$ which entails that $A$ has finite fractal dimension. Medjo in [7] studied the pullback asymptotic behavior of solutions for a non-autonomous homogeneous two-phase flow model in a two-dimensional domain.

Recently the appearance of delay effects in partial difference equations has been intensively treated. In [8-10], the authors studied the 2D Navier-Stokes equations in which additional external forces were included in the model. The existence of an attractor for a 2D Navier-Stokes system with delays is proved in [8]. The authors proved the existence and uniqueness of a stationary solution and the exponential decay of the solutions of the evolutionary problem to this stationary solution in [9] and strengthened some results on the existence and properties of pullback attractors in [10]. It is a natural generalization to the Allen-Cahn-Navier-Stokes equations with delays. Medjo in [11, 12] studied a coupled Cahn-Hilliard-Navier-Stokes model with delays in a two-dimensional domain and proved

(c) The Author(s) 2017. This article is distributed under the terms of the Creative Commons Attribution 4.0 International License (http://creativecommons.org/licenses/by/4.0/), which permits unrestricted use, distribution, and reproduction in any medium, provided you give appropriate credit to the original author(s) and the source, provide a link to the Creative Commons license, and indicate if changes were made. 
the existence and uniqueness of the weak and strong solution when the external force contains some delays. He also discusses the asymptotic behavior of the weak solutions and the stability of the stationary solutions.

Our purpose is to study a coupled Allen-Cahn-Navier-Stokes model with infinite delays. The paper is organized as follows. Section 2 we describe the model and some functional spaces useful for the problem. Section 3 we prove the existence and uniqueness of the solution. Also we analyze the continuity properties of the solutions with respect to initial data. Section 4 is devoted to generalizing the results on asymptotic behavior, proving under more general assumptions the existence of pullback attractors.

\section{A two-phase flow model}

We consider a model of homogeneous incompressible two-phase flow with singularly oscillating forces. We assume that the domain $\Omega \subset R^{2}$ be an open and bounded set with smooth enough boundary $\partial \Omega$ and consider (arbitrary) values $\tau<T$ in $R$. Then we consider the following system:

$$
\left\{\begin{array}{l}
\frac{\partial u}{\partial t}-v \Delta \boldsymbol{u}+(\boldsymbol{u} \cdot \nabla) \boldsymbol{u}+\nabla p-\mathcal{K} \mu \nabla \phi=\boldsymbol{g}(t)+G\left(t,(\boldsymbol{u}, \phi)_{t}\right) \\
\operatorname{div} \boldsymbol{u}=0 \\
\frac{\partial \phi}{\partial t}+\boldsymbol{u} \cdot \nabla \phi+\mu=0 \\
\mu=-\epsilon \Delta \phi+\alpha f(\phi),
\end{array}\right.
$$

in $\Omega \times(\tau, T)$.

In (1), the unknown functions are the velocity $\boldsymbol{u}=\left(u_{1}, u_{2}\right)$ of the fluid, its pressure $p$ and the order parameter $\phi$. The quantity $\mu$ is the variational derivative of the following free energy functional:

$$
\mathcal{F}(\phi)=\int_{\Omega}\left(\frac{\epsilon}{2}|\nabla \phi|^{2}+\alpha F(\phi)\right) d s
$$

where $F(r)=\int_{0}^{r} f(\zeta) d \zeta$, the constants $v>0$ and $\mathcal{K}>0$ the kinematic viscosity of the fluid and capillarity coefficient, respectively, $\epsilon, \alpha>0$ are two physical parameters describing the interaction between the two phases. In particular, $\epsilon$ is related with the thickness of the interface separating the two fluid. The number $\tau \in R$ is the initial time. We endow (1) with the boundary condition

$$
\boldsymbol{u}=0, \quad \frac{\partial \phi}{\partial \eta}=0 \quad \text { on } \partial \Omega \times(\tau, T)
$$

where $\partial \Omega$ is the boundary of $\Omega$ and $\eta$ is its outward normal. The initial condition is given by

$$
(\mathbf{u}, \phi)(t+\tau)=\vartheta(t)=\left(\vartheta_{1}, \vartheta_{2}\right)(t), \quad t \in(-\infty, 0]
$$

The terms $\boldsymbol{g}(t)$ is a non-delayed external force field, $G\left(t,(\boldsymbol{u}, \phi)_{t}\right)$ is another external force containing some hereditary characteristics and we denote by $(\boldsymbol{u}, \phi)_{t}$ the function defined on $(-\infty, 0]$ by the relation $(\boldsymbol{u}, \phi)_{t}=(\boldsymbol{u}, \phi)(t+s), s \in(-\infty, 0]$. 
We assume that $f \in C^{1}(R)$ satisfies

$$
\lim _{|r| \rightarrow+\infty} f^{\prime}(r)>0, \quad\left|f^{\prime}(r)\right| \leq c_{f}\left(1+|r|^{k}\right), \quad \forall r \in R
$$

where $c_{f}$ is some positive constant and $k \in[1,+\infty)$ is fixed. So we get

$$
|f(r)| \leq c_{f}\left(1+|r|^{k+1}\right), \quad \forall r \in R
$$

If $X$ is a real Hilbert space with inner product $(\cdot, \cdot)_{X}$, we will denote the induced norm by $|\cdot|_{X}$ while $X^{*}$ will indicate its dual with $\langle\cdot, \cdot\rangle$ for the duality between $X^{*}$ and $X$ and the norm by $\|\cdot\|_{*}$. We set

$$
\mathcal{V}_{1}=\left\{\boldsymbol{u} \in C_{0}^{\infty}(\Omega): \operatorname{div} \boldsymbol{u}=0 \text { in } \Omega\right\}
$$

We denote by $H_{1}$ and $V_{1}$ the closure of $\mathcal{V}_{1}$ in $\left(L^{2}(\Omega)\right)^{2}$ and $\left(H_{0}^{1}(\Omega)\right)^{2}$, respectively. The scalar product in $H_{1}$ is denoted by $(\cdot, \cdot)_{L^{2}}$ and the associated norm by $|\cdot|_{L^{2}}$. Moreover, the space $V_{1}$ is endowed with the scalar product

$$
((\boldsymbol{u}, \boldsymbol{v}))=\sum_{i=1}^{2}\left(\partial_{x_{i}} \boldsymbol{u}, \partial_{x_{i}} \boldsymbol{v}\right)_{L^{2}}, \quad\|\boldsymbol{u}\|=((\boldsymbol{u}, \boldsymbol{u}))^{1 / 2}
$$

We define the operator $\boldsymbol{A}_{0}$ by

$$
\boldsymbol{A}_{0} u=P \Delta \boldsymbol{u}, \quad \forall \boldsymbol{u} \in D\left(\boldsymbol{A}_{0}\right)=H^{2}(\Omega) \cap V_{1},
$$

where $P$ is the Leray-Helmholtz projector from $L^{2}(\Omega)$ onto $H_{1}$. Then $\boldsymbol{A}_{0}$ is a self-adjoint positive unbounded operator in $H_{1}$ which is associated with the scalar product defined above. Furthermore, $\boldsymbol{A}_{0}^{-1}$ is a compact linear operator on $H_{1}$ and $\left|\boldsymbol{A}_{0} \cdot\right|_{L^{2}}$ is a norm on $D\left(\boldsymbol{A}_{0}\right)$ that is equivalent to the $H^{2}$-norm. From (3), we can find $\gamma>0$ such that

$$
\lim _{|r| \rightarrow+\infty} f^{\prime}(r)>2 \gamma>0
$$

Then we can define the linear positive unbounded operator $A_{\gamma}$ on $L^{2}(\Omega)$ by

$$
A_{\gamma} \phi=-\Delta \phi+\gamma \phi, \quad \forall \phi \in D\left(A_{\gamma}\right)
$$

where

$$
D\left(A_{\gamma}\right)=\left\{\rho \in H^{2}(\Omega) ; \frac{\partial \rho}{\partial \eta}=0 \text { on } \partial \Omega\right\} .
$$

Note that $A_{\gamma}^{-1}$ is a compact linear operator on $L^{2}(\Omega)$ and $\left|A_{\gamma} \cdot\right|_{L^{2}}$ is a norm on $D\left(A_{\gamma}\right)$ that is equivalent to the $H^{2}$-norm. We set $V_{2}=H^{1}(\Omega)$. Furthermore we denote by $\lambda_{1}>0$ a positive constant satisfying

$$
\lambda_{1}|\omega|_{L^{2}}^{2} \leq\|\omega\|^{2} \quad \forall \omega \in V_{1} ; \quad \lambda_{1}\left|A_{\gamma}^{1 / 2} \psi\right|_{L^{2}}^{2} \leq\left|A_{\gamma} \psi\right|_{L^{2}}^{2} \quad \forall \psi \in H^{2}(\Omega) .
$$


We also introduce the bilinear operators $B_{0}, B_{1}$ and their associated trilinear forms $b_{0}$, $b_{1}$ as well as the coupling mapping $\boldsymbol{R}_{0}$, which are defined from $D\left(\boldsymbol{A}_{0}\right) \times D\left(\boldsymbol{A}_{0}\right)$ into $H_{1}$, $D\left(\boldsymbol{A}_{0}\right) \times D\left(A_{\gamma}\right)$ into $L_{2}(\Omega)$ and $L^{2}(\Omega) \times D\left(A_{\gamma}^{3 / 2}\right)$ into $H_{1}$, respectively. More precisely, we set

$$
\begin{aligned}
& \left(B_{0}(\boldsymbol{u}, \boldsymbol{v}), \boldsymbol{w}\right)=\int_{\Omega}[(\boldsymbol{u} \cdot \nabla) \boldsymbol{v}] \cdot \boldsymbol{w} d x=b_{0}(\boldsymbol{u}, \boldsymbol{v}, \boldsymbol{w}), \quad \forall \mathbf{u}, \boldsymbol{v}, \boldsymbol{w} \in D\left(\boldsymbol{A}_{0}\right), \\
& \left(B_{1}(\boldsymbol{u}, \phi), \rho\right)=\int_{\Omega}[(\boldsymbol{u} \cdot \nabla) \phi] \rho d x=b_{1}(\boldsymbol{u}, \phi, \rho), \quad \forall \boldsymbol{u} \in D\left(\boldsymbol{A}_{0}\right), \phi, \rho \in D\left(A_{\gamma}\right), \\
& \left(\boldsymbol{R}_{0}(\mu, \phi), \boldsymbol{w}\right) \\
& \quad=\int_{\Omega} \mu[\nabla \phi \cdot \boldsymbol{w}] d x=b_{1}(\boldsymbol{w}, \phi, \mu), \quad \forall \boldsymbol{w} \in D\left(A_{0}\right),(\mu, \phi) \in L^{2}(\Omega) \times D\left(A_{\gamma}^{3 / 2}\right) .
\end{aligned}
$$

Note that $\boldsymbol{R}_{0}(\mu, \phi)=P_{1} \mu \nabla \phi$ and $B_{0}, B_{1}$ and $\boldsymbol{R}_{0}$ satisfy the following estimates:

$$
\begin{aligned}
& \left|B_{0}(\boldsymbol{u}, \boldsymbol{v})\right|_{V_{1}^{*}} \leq c|\boldsymbol{u}|_{L^{2}}^{1 / 2}\|\boldsymbol{u}\|^{1 / 2}|\boldsymbol{v}|_{L^{2}}^{1 / 2}\|\boldsymbol{v}\|^{1 / 2}, \quad \forall \boldsymbol{u}, \boldsymbol{v} \in V_{1}, \\
& \left|B_{1}(\boldsymbol{u}, \phi)\right|_{V_{2}^{*}} \leq c|\boldsymbol{u}|_{L^{2}}^{1 / 2}\|\boldsymbol{u}\|^{1 / 2}|\phi|_{L^{2}}^{1 / 2}\|\phi\|^{1 / 2}, \quad \forall \boldsymbol{u} \in V_{1}, \phi \in V_{2}, \\
& \left|\boldsymbol{R}_{0}\left(A_{\gamma} \phi, \rho\right)\right|_{V_{1}^{*}} \leq c\|\rho\|^{1 / 2}\left|A_{\gamma} \rho\right|_{L^{2}}^{1 / 2}\left|A_{\gamma} \phi\right|_{L^{2}}, \quad \forall \phi, \rho \in D\left(A_{\gamma}\right) .
\end{aligned}
$$

Now we define the Hilbert spaces $\mathbb{Y}$ and $\mathbb{V}$ by $\mathbb{Y}=H_{1} \times H^{1}(\Omega), \mathbb{V}=V_{1} \times D\left(A_{\gamma}\right)$ endowed with the scalar products whose associated norms are

$$
\begin{aligned}
& |(\boldsymbol{u}, \phi)|_{\mathbb{Y}}^{2}=\mathcal{K}^{-1}|\boldsymbol{u}|_{L^{2}}^{2}+\epsilon\left(|\nabla \phi|_{L^{2}}^{2}+\gamma|\phi|_{L^{2}}^{2}\right)=\mathcal{K}^{-1}|\boldsymbol{u}|_{L^{2}}^{2}+\epsilon\left|A_{\gamma}^{1 / 2} \phi\right|_{L^{2}}^{2} ; \\
& \|(\boldsymbol{u}, \phi)\|_{\mathbb{V}}^{2}=\|\boldsymbol{u}\|^{2}+\left|A_{\gamma} \phi\right|_{L^{2}}^{2} .
\end{aligned}
$$

Let $f_{\gamma}(r)=f(r)-\alpha^{-1} \epsilon \gamma r$ and observe that $f_{\gamma}$ still satisfies (5) with $\gamma$ in place of $2 \gamma$ since $\epsilon \leq \alpha$, and $F_{\gamma}(r)=\int_{0}^{r} f_{\gamma}(\zeta) d \zeta$ is bounded from below.

There are several phase spaces which allow us to deal with infinite delays. For instance, for a given $\delta>0$, and a given Banach space $X$, we may consider the space

$$
C_{\delta}(X)=\left\{\varphi \in C((-\infty, 0] ; X): \exists \lim _{s \rightarrow-\infty} e^{\delta s} \varphi(s) \in X\right\}
$$

with the norm

$$
\|\varphi\|_{\delta}:=\sup _{s \in(-\infty, 0]} e^{\delta s}|\varphi(s)|_{X}
$$

In order to state the problem in the correct framework, we assume that $G:[\tau, T] \times$ $C_{\delta}(\mathbb{Y}) \rightarrow\left(L^{2}(\Omega)\right)^{2}$ satisfies

(g1) for any $(\boldsymbol{u}, \phi) \in C_{\delta}(\mathbb{Y}), t \in[\tau, T] \rightarrow G(t,(\boldsymbol{u}, \phi))$ is measurable;

(g2) for any $t \in R, G(t, 0)=0$;

(g3) there exists a constant $L_{g}>0$ such that, for any $t \in[\tau, T],\left(\boldsymbol{u}_{1}, \phi_{1}\right),\left(\boldsymbol{u}_{2}, \phi_{2}\right) \in C_{\delta}(\mathbb{Y})$,

$$
\left|G\left(t,\left(\boldsymbol{u}_{1}, \phi_{1}\right)\right)-G\left(t,\left(\boldsymbol{u}_{2}, \phi_{2}\right)\right)\right|_{L^{2}} \leq L_{g}\left\|\left(\boldsymbol{u}_{1}, \phi_{1}\right)-\left(\boldsymbol{u}_{2}, \phi_{2}\right)\right\|_{\delta} .
$$

An example of an operator satisfying assumptions (g1)-(g3) is given here. 
We will assume that the distributed delay term on the Banach space $C_{\delta}(\mathbb{Y})$ is given by

$$
G\left(t,(\boldsymbol{u}, \phi)_{t}\right):=\int_{-\infty}^{0} G_{0}(t, s,(\boldsymbol{u}, \phi)(t+s)) d s
$$

where the function $G_{0}:[\tau, T] \times(-\infty, 0] \times \mathbb{Y} \rightarrow\left(L^{2}(\Omega)\right)^{2}$ satisfies

$$
G_{0}(t, s, 0)=0, \quad\left|G_{0}\left(t, s,\left(\boldsymbol{u}, \phi_{1}\right)\right)-G_{0}\left(t, s,\left(\boldsymbol{u}_{2}, \phi_{2}\right)\right)\right|_{R^{2}} \leq L_{1}(s)\left|\left(\boldsymbol{u}_{1}, \phi_{1}\right)-\left(\boldsymbol{u}_{2}, \phi_{2}\right)\right|_{\mathbb{Y}},
$$

for the function $L_{1}>0$. We assume that $L_{1}(\cdot) e^{-(\delta+\theta) \cdot} \in L^{2}(-\infty, 0)$ for certain $\theta>0$. We can prove as in [9] that (g1)-(g3) are all satisfied.

Hereafter, we will use the above space and the distributed delay for our problem.

Using the notations above, we rewrite (1) in the form

$$
\left\{\begin{array}{l}
\frac{d \boldsymbol{u}}{d t}+v A_{0} \boldsymbol{u}+B_{0}(\boldsymbol{u}, \boldsymbol{u})-\mathcal{K} \boldsymbol{R}_{0}\left(\epsilon A_{\gamma} \phi, \phi\right)=\boldsymbol{g}(t)+G\left(t,(\boldsymbol{u}, \phi)_{t}\right), \\
\frac{d \phi}{d t}+B_{1}(\boldsymbol{u}, \phi)+\mu=0, \\
\mu=\epsilon A_{\gamma} \phi+\alpha f_{\gamma}(\phi), \\
(\boldsymbol{u}, \phi)(t+\tau)=\vartheta(t)=\left(\vartheta_{1}, \vartheta_{2}\right)(t), \quad t \in(-\infty, 0] .
\end{array}\right.
$$

Remark 1 In the weak formulation (10), the term $\mu \nabla \phi$ is replaced by $\epsilon A_{\gamma} \nabla \phi$. This is justified since $f_{\gamma}^{\prime}(\phi)$ is the gradient $F_{\gamma}(\phi)$ and can be incorporated into the pressure gradient; see [10]. To simplify the notation, we set $\alpha=\mathcal{K}=1$.

Remark 2 Set $\forall(\omega, \psi) \in \mathbb{Y}$

$$
E(t)=\varepsilon(\omega, \psi)=|(\omega, \psi)|_{\mathbb{Y}}^{2}+2\left\langle F_{\gamma}(\psi), 1\right\rangle_{L^{2}}+\alpha_{0}
$$

where $\alpha_{0}$ is a constant large enough and independent on $(\omega, \psi)$ such that $E(t)$ is nonnegative.

Definition 1 A pair $(\boldsymbol{u}, \phi)$ is called a weak solution to (10) if

$$
\begin{aligned}
& (\boldsymbol{u}, \phi) \in C((-\infty, T] ; \mathbb{Y}) \cap L^{2}([\tau, T] ; \mathbb{V}), \quad \frac{d \boldsymbol{u}}{d t} \in L^{1}\left([\tau, T] ; V_{1}^{*}\right), \\
& \frac{d \phi}{d t}, \mu \in L^{1}\left([\tau, T] ; V_{2}^{*}\right),
\end{aligned}
$$

and $(\boldsymbol{u}, \phi)$ satisfies $(10)_{1}$ and $(10)_{3}$ in $V_{1}^{*}$ and $V_{2}^{*}$, respectively.

Remark 3 If $(\boldsymbol{u}, \phi)$ is a weak solution of (10) in the sense given above, then $(\boldsymbol{u}, \phi)$ satisfies an energy equality. Namely,

$$
\begin{aligned}
E(t) & -E(s)+\int_{s}^{t}\left(2 v\|\boldsymbol{u}\|^{2}+2|\mu|_{L^{2}}^{2}\right) d r \\
& =2 \int_{s}^{t}\left(\langle\boldsymbol{g}(r), \boldsymbol{u}(r)\rangle+\left\langle G\left(r,(\boldsymbol{u}, \phi)_{r}\right), \boldsymbol{u}(r)\right\rangle\right) d r \quad \forall s, t \in[\tau, T],
\end{aligned}
$$

where $E(t)=\varepsilon(\boldsymbol{u}, \phi)$. 


\section{Existence of solutions}

In this section we establish existence of weak and strong solution for problem (1) as additional assumptions are satisfied and some related properties.

Theorem 1 Assume that $\boldsymbol{g} \in L^{2}\left(\tau, T ; V_{1}^{*}\right), G:[\tau, T] \times C_{\delta}(\mathbb{Y}) \rightarrow\left(L^{2}(\Omega)\right)^{2}$ satisfies (g1)-(g3). Then, for $\vartheta \in C_{\delta}(\mathbb{Y})$, there exists a unique weak solution $(\boldsymbol{u}, \phi)$ of $(10)$.

Proof For the existence, we split the proof into several steps.

Step 1: A Galerkin scheme. Since the injection of $\mathbb{V} \subset \mathbb{Y}$ is compact. Let $\left\{\left(\omega_{i}, \psi_{i}\right), i=\right.$ $1,2,3, \ldots\} \subset \mathbb{V}$ be an orthonormal basis of $\mathbb{Y}$, where $\left\{\omega_{i}, i=1,2, \ldots\right\},\left\{\psi_{i}, i=1,2, \ldots\right\}$ are eigenvectors of $\boldsymbol{A}_{0}$ and $A_{\gamma}$, respectively. We set $\mathbb{V}_{m}=\mathbb{Y}_{m}=\operatorname{span}\left\{\left(\omega_{1}, \psi_{1}\right),\left(\omega_{2}, \psi_{2}\right), \ldots\right.$, $\left.\left(\boldsymbol{\omega}_{m}, \psi_{m}\right)\right\}$. We look for $\left(\boldsymbol{u}^{m}, \phi^{m}\right) \in \mathbb{Y}_{m}$, the solution to the ordinary differential equations

$$
\left\{\begin{array}{l}
\frac{d \mathbf{u}^{m}}{d t}+P_{1}^{m}\left(v \boldsymbol{A}_{0} \mathbf{u}^{m}+B_{0}\left(\boldsymbol{u}^{m}, \boldsymbol{u}^{m}\right)-\boldsymbol{R}_{0}\left(\epsilon A_{\gamma} \phi^{m}, \phi^{m}\right)\right) \\
\quad=P_{1}^{m}\left(\boldsymbol{g}(t)+G\left(t,\left(\boldsymbol{u}^{m}, \phi^{m}\right) t\right)\right) \\
\frac{d \phi^{m}}{d t}+P_{2}^{m}\left(B_{1}\left(\boldsymbol{u}^{m}, \phi^{m}\right)+\mu^{m}\right)=0 \\
\mu^{m}=P_{2}^{m}\left(\epsilon A_{\gamma} \phi^{m}+f_{\gamma}\left(\phi^{m}\right)\right) \\
\left(\boldsymbol{u}^{m}, \phi^{m}\right)(t+\tau)=P^{m} \vartheta(t), \quad t \in(-\infty, 0]
\end{array}\right.
$$

where $P^{m}=\left(P_{1}^{m}, P_{2}^{m}\right): H_{1} \times L^{2}(\Omega) \rightarrow \mathbb{V}^{m}$ is the orthogonal projection. Since $P^{m}(0, G(t$, $\left.\left.(\boldsymbol{u}, \phi)_{t}\right)\right)$ is a local Lipschitz function in $(\boldsymbol{u}, \phi)$, it follows from the theory of ordinary differential equations that this equation has a local solution $\left(\boldsymbol{u}^{m}, \phi^{m}\right)$.

Next we will deduce $a$ priori estimates that ensure that the solutions do exist for all time.

Step 2: A priori estimates. By taking the scalar product in $H_{1}$ of $(11)_{1}$ with $\boldsymbol{u}^{m}$, then taking the scalar product in $L^{2}(\Omega)$ of $(11)_{3}$ with $\mu^{m}$, we get

$$
\frac{d E^{m}}{d t}+2 v\left\|\boldsymbol{u}^{m}\right\|^{2}+2\left|\mu^{m}\right|_{L^{2}}^{2}=2\left\langle\boldsymbol{g}(t), \boldsymbol{u}^{m}\right\rangle+2\left\langle G\left(t,\left(\boldsymbol{u}^{m}, \phi^{m}\right)_{t}\right), \boldsymbol{u}^{m}\right\rangle,
$$

where $E^{m}(t)=\varepsilon\left(\boldsymbol{u}^{m}(t), \phi^{m}(t)\right)$. Then by (g3), we have

$$
\begin{aligned}
\frac{d E^{m}}{d t}+2 v\left\|\boldsymbol{u}^{m}\right\|^{2}+2\left|\mu^{m}\right|_{L^{2}}^{2} & \leq 2\|\boldsymbol{g}(t)\|_{*}\left\|\boldsymbol{u}^{m}\right\|+2 L_{g}\left\|\left(\boldsymbol{u}^{m}, \phi^{m}\right)_{t}\right\|_{\delta}\left|\boldsymbol{u}^{m}\right|_{L^{2}} \\
& \leq v\left\|\boldsymbol{u}^{m}\right\|^{2}+v^{-1}\|\boldsymbol{g}(t)\|_{*}^{2}+2 L_{g}\left\|\left(\boldsymbol{u}^{m}, \phi^{m}\right)_{t}\right\|_{\delta}^{2} ;
\end{aligned}
$$

then

$$
\begin{aligned}
\frac{d E^{m}}{d t}+v\left\|\boldsymbol{u}^{m}\right\|^{2}+2\left|\mu^{m}\right|_{L^{2}}^{2} & \leq v^{-1}\|\boldsymbol{g}(t)\|_{*}^{2}+2 L_{g}\left\|\left(\boldsymbol{u}^{m}, \phi^{m}\right)_{t}\right\|_{\delta}^{2} \\
& \leq v^{-1}\|\boldsymbol{g}(t)\|_{*}^{2}+2 L_{g}\left\|E_{t}^{m}\right\|_{\delta},
\end{aligned}
$$

where

$$
\|E\|_{\delta}=\sup _{s \in(-\infty, 0]} e^{2 \delta s} E(s)
$$

and therefore

$$
E^{m}(t)+\int_{\tau}^{t}\left(v\left\|\boldsymbol{u}^{m}\right\|^{2}+2\left|\mu^{m}\right|_{L^{2}}^{2}\right) d s \leq E(\tau)+\int_{\tau}^{t}\left(v^{-1}\|\boldsymbol{g}(s)\|_{*}^{2}+2 L_{g}\left\|E_{s}^{m}\right\|_{\delta}\right) d s .
$$


Thus

$$
\begin{aligned}
\left\|E_{t}^{m}\right\|_{\delta} \leq & \max \left\{\sup _{\theta \in(-\infty, \tau-t]} e^{2 \delta \theta} E^{m}(\vartheta(t+\theta-\tau)),\right. \\
& \left.\sup _{\theta \in[\tau-t, 0]}\left(e^{2 \delta \theta} E(\tau)+e^{2 \delta \theta} \int_{\tau}^{t+\theta}\left(v^{-1}\|\boldsymbol{g}(s)\|_{*}^{2}+2 L_{g}\left\|E_{s}^{m}\right\|_{\delta}\right) d s\right)\right\} \\
\leq & \max \left\{\sup _{\theta \in(-\infty, \tau-t]} e^{2 \delta \theta} E^{m}(\vartheta(t+\theta-\tau)),\right. \\
& \left.E(\tau)+\int_{\tau}^{t}\left(v^{-1}\|\boldsymbol{g}(s)\|_{*}^{2}+2 L_{g}\left\|E_{s}^{m}\right\|_{\delta}\right) d s\right\}
\end{aligned}
$$

and

$$
\begin{aligned}
\sup _{\theta \in(-\infty, \tau-t]} e^{2 \delta \theta} E^{m}(\vartheta(t+\theta-\tau)) & =\sup _{\theta \leq 0} e^{2 \delta(\theta-(t-\tau))} E(\vartheta(\theta)) \\
& =e^{-2 \delta(t-\tau)}\|E(\vartheta)\|_{\delta} \leq\|E(\vartheta)\|_{\delta}
\end{aligned}
$$

and $E(\tau)=E(\vartheta(0)) \leq\|E(\vartheta)\|_{\delta}$, so we can obtain

$$
\left\|E_{t}^{m}\right\|_{\delta} \leq\|E(\vartheta)\|_{\delta}+\int_{\tau}^{t}\left(v^{-1}\|\boldsymbol{g}(s)\|_{*}^{2}+2 L_{g}\left\|E_{s}^{m}\right\|_{\delta}\right) d s .
$$

Thus by Gronwall's lemma, we have

$$
\left\|E_{t}^{m}\right\|_{\delta} \leq e^{2 L_{g}(t-\tau)}\left(\|E(\vartheta)\|_{\delta}+\int_{\tau}^{t} v^{-1}\|\boldsymbol{g}(s)\|_{*}^{2} d s\right) .
$$

Using this inequality, we also see that there exists a constant $C$, depending on some constants of the problem (namely, $v, L_{g}$ and $\boldsymbol{g}$ ) and on $\tau, T$ and $R>0$, such that

$$
\left\|E_{t}^{m}\right\|_{\delta} \leq C(\tau, T, R), \quad E^{m}(t) \leq C(\tau, T, R) .
$$

As $\left|\left(\boldsymbol{u}^{m}, \phi^{m}\right)\right|_{\mathbb{Y}}^{2} \leq E^{m}(t)$, this implies that $\left(\boldsymbol{u}^{m}, \phi^{m}\right)$ is bounded in $L^{\infty}(\tau, T ; \mathbb{Y}) \cap L^{2}(\tau, T, \mathbb{V})$ $\forall T>\tau$. Noting that

$$
\mu^{m}=\epsilon A_{\gamma} \phi^{m}+\alpha f_{\gamma}\left(\phi^{m}\right)
$$

we get [6]

$$
\begin{aligned}
& \left|A_{\gamma} \phi^{m}\right|_{L^{2}} \leq c\left|\mu^{m}\right|_{L^{2}}+Q_{1}\left(\left\|\phi^{m}\right\|\right), \\
& \left|A_{\gamma}^{3 / 2} \phi^{m}\right|_{L^{2}} \leq c\left|\mu^{m}\right|_{L^{2}}+Q_{1}\left(\left\|\phi^{m}\right\|\right),
\end{aligned}
$$

where $Q_{1}$ is a monotone nondecreasing function independent on time, the initial condition and $m$. Then we see that $A_{\gamma}^{3 / 2} \phi^{m}$ is bounded in $L^{2}\left(\tau, T ; D\left(A_{\gamma}^{3 / 2}\right)\right)$. With (7)-(9), (15) and (16) we get

$$
\frac{d}{d t}\left(\boldsymbol{u}^{m}, \phi^{m}\right) \quad \text { is bounded in } L^{2}\left(\tau, T ; V_{1}^{*}\right) \times L^{2}\left(\tau, T ; V_{2}^{*}\right)
$$


Step 3: Approximation in $C_{\delta}(\mathbb{Y})$ of the initial datum. For the initial datum $\vartheta \in C_{\delta}(\mathbb{Y})$ we have used the projections in the Galerkin scheme in Step 1. Let us check that

$$
P^{m} \vartheta \rightarrow \vartheta \quad \text { in } C_{\delta}(\mathbb{Y})
$$

Indeed assume there do not exist $\epsilon>0$ and a subsequence such that

$$
e^{\delta \theta_{m}}\left|P^{m} \vartheta\left(\theta_{m}\right)-\vartheta\left(\theta_{m}\right)\right|>\epsilon
$$

One can assume that $\theta_{m} \rightarrow-\infty$. Otherwise, if $\theta_{m} \rightarrow \theta$ then $P^{m} \vartheta\left(\theta_{m}\right) \rightarrow \vartheta(\theta)$. We have $\left|P^{m} \vartheta\left(\theta_{m}\right)-\vartheta(\theta)\right| \leq\left|P^{m} \vartheta\left(\theta_{m}\right)-P^{m} \vartheta(\theta)\right|+\left|P^{m} \vartheta(\theta)-\vartheta(\theta)\right| \rightarrow 0$ as $m \rightarrow+\infty$. But with $\theta_{m} \rightarrow-\infty$ as $m \rightarrow+\infty$ if we denote $x=\lim _{\theta \rightarrow-\infty} e^{\delta \theta} \vartheta(\theta)$, we obtain

$$
\begin{aligned}
e^{\delta \theta_{m}}\left|P^{m} \vartheta\left(\theta_{m}\right)-\vartheta\left(\theta_{m}\right)\right| & =\left|P^{m}\left(e^{\delta \theta_{m}} \vartheta\left(\theta_{m}\right)\right)-e^{\delta \theta_{m}} \vartheta\left(\theta_{m}\right)\right| \\
& \leq\left|P^{m}\left(e^{\delta \theta_{m}} \vartheta\left(\theta_{m}\right)\right)-P^{m} x\right|+\left|P^{m} x-x\right|+\left|x-e^{\delta \theta_{m}} \vartheta\left(\theta_{m}\right)\right| \\
& \rightarrow 0 .
\end{aligned}
$$

Thus there is a contradiction.

Step 4: Energy method and compactness results. Now using the standard methods as in [13], we can pass to the limit in (11) as $m \rightarrow \infty$ and we see that $(\boldsymbol{u}, \phi)$ is a weak solution of (11).

From Step 2 we get a subsequence (still) denoted by $\left(\boldsymbol{u}^{m}, \phi^{m}\right)$ and using the compactness theorem

$$
\left\{\begin{array}{l}
\left(\boldsymbol{u}^{m}, \phi^{m}\right) \stackrel{*}{\rightarrow}(\boldsymbol{u}, \phi) \quad \text { weakly star in } L^{\infty}(\tau, T ; \mathbb{Y}), \\
\left(\boldsymbol{u}^{m}, \phi^{m}\right) \rightarrow(\boldsymbol{u}, \phi) \quad \text { weakly in } L^{2}(\tau, T ; \mathbb{V}), \\
\frac{d}{d t}\left(\boldsymbol{u}^{m}, \phi^{m}\right) \rightarrow \frac{d}{d t}(\boldsymbol{u}, \phi) \quad \text { weakly in } L^{2}\left(\tau, T ; V_{1}^{*}\right) \times L^{2}\left(\tau, T ; V_{2}^{*}\right), \\
\left(\boldsymbol{u}^{m}, \phi^{m}\right) \rightarrow(\boldsymbol{u}, \phi) \quad \text { strongly in } L^{2}(\tau, T ; \mathbb{Y}), \\
G\left(\cdot,\left(\boldsymbol{u}^{m}, \phi\right)\right) \rightarrow \zeta \quad \text { weakly in } L^{2}\left(\tau, T ;\left(L^{2}(\Omega)\right)^{2}\right),
\end{array}\right.
$$

for all $T>\tau$. Furthermore we can also assume that

$$
\left(\boldsymbol{u}^{m}, \phi^{m}\right)(t) \rightarrow(\boldsymbol{u}, \phi)(t) \quad \text { in } \mathbb{Y} \text { a.e. } t \in(\tau, T)
$$

which nevertheless is not enough.

Since the injection of $\mathbb{V}$ into $\mathbb{Y}$ is compact, the injection of $\mathbb{Y}$ into $\mathbb{V}^{*}$ is compact too. So by the Ascoli-Arzela theorem we have

$$
\left(\boldsymbol{u}^{m}, \phi^{m}\right)(t) \rightarrow(\boldsymbol{u}, \phi)(t) \quad \text { in } C\left([\tau, T], \mathbb{V}^{*}\right)
$$

Then, for any $\left\{t_{m}\right\} \subset[\tau, T]$, with $t_{m} \rightarrow t$, we have

$$
\left(\boldsymbol{u}^{m}, \phi^{m}\right)\left(t_{m}\right) \rightarrow\left(\boldsymbol{u}^{m}, \phi^{m}\right)(t) \quad \text { weakly in } \mathbb{Y}
$$


and

$$
E^{m}\left(t_{m}\right) \rightarrow E(t) \quad \text { weakly in } \mathbb{Y} .
$$

Next we will prove that

$$
\left(\boldsymbol{u}^{m}, \phi^{m}\right)\left(t_{m}\right) \rightarrow\left(\boldsymbol{u}^{m}, \phi^{m}\right)(t) \quad \text { in } C([\tau, T] ; \mathbb{Y}) .
$$

That is, we will prove that

$$
E^{m}\left(t_{m}\right) \rightarrow E(t) \quad \text { in } C([\tau, T] ; \mathbb{Y}) .
$$

If this were not so we take into account that $(\boldsymbol{u}, \phi) \in C([\tau, T] ; \mathbb{Y})$, there would exist $\epsilon>0$ and $t_{0} \in[\tau, T]$ and subsequences (relabeled the same) $\left\{\left(\boldsymbol{u}^{m}, \phi^{m}\right)\right\}$ and $\left\{t_{m}\right\} \subset[\tau, T]$ with $\lim _{m \rightarrow+\infty} t_{m}=t_{0}$ such that

$$
\left|E^{m}\left(t_{m}\right)-E\left(t_{0}\right)\right| \geq \epsilon \quad \forall m \text {. }
$$

To prove that this is absurd, we will use the energy method. Observe that the following energy inequality holds for all $\left(\boldsymbol{u}^{m}, \phi^{m}\right)$ :

$$
\begin{aligned}
& \frac{1}{2} E^{m}(t)+\frac{1}{2} \int_{s}^{t}\left(v\left\|\boldsymbol{u}^{m}(r)\right\|^{2}+\left|\mu^{m}\right|_{L^{2}}\right) d r \\
& \leq \int_{s}^{t}\left\langle\boldsymbol{g}(r), \boldsymbol{u}^{m}(r)\right\rangle d r+\frac{1}{2} E^{m}(s)+C(t-s) \quad \forall s, t \in[\tau, T],
\end{aligned}
$$

where $C=\frac{D}{2 v \lambda_{1}}$ and $D$ corresponds to the upper bound

$$
\int_{s}^{t}\left|G\left(r,\left(\boldsymbol{u}^{m}, \phi^{m}\right)_{r}\right)\right|^{2} d r \leq D(t-s), \quad \tau \leq s \leq t \leq T
$$

by (g2), (g3) and (12).

On the other hand, by (17), passing to the limit in (11), we see that $(\boldsymbol{u}, \phi) \in C([\tau, T] ; \mathbb{Y})$ is a solution of a similar problem to (10),

$$
\frac{d E}{d t}+2 v\|\boldsymbol{u}\|^{2}+2|\mu|_{L^{2}}^{2}=2\langle\boldsymbol{g}(t), \boldsymbol{u}\rangle+2(\zeta, \boldsymbol{u})
$$

with the initial data $(\boldsymbol{u}, \phi)(\tau)=\vartheta(0)$. Therefore, it satisfies the energy equality

$$
\begin{aligned}
E(t) & +\int_{t}^{s}\left(2 v\|\boldsymbol{u}\|^{2}+2|\mu|_{L^{2}}^{2}\right) d r \\
& =E(s)+2 \int_{s}^{t}\left(\langle\boldsymbol{g}(r), \boldsymbol{u}(r)\rangle+\left\langle G\left(r,(\boldsymbol{u}, \phi)_{r}\right), \boldsymbol{u}(r)\right\rangle\right) d r \quad \forall s, t \in[\tau, T] .
\end{aligned}
$$

Furthermore, from the last convergence in (17), we deduce that

$$
\int_{s}^{t}|\zeta(r)|^{2} d r \leq \lim _{m \rightarrow+\infty} \sup \int_{s}^{t}\left|G\left(r,(\boldsymbol{u}, \phi)_{r}^{m}\right)\right|^{2} d r \leq D(t-s) \quad \forall \tau \leq s \leq t \leq T .
$$


Now consider the functions $J_{m}, J:[\tau, T] \rightarrow R$ defined by

$$
\begin{aligned}
& J_{m}(t)=\frac{1}{2} E^{m}(t)-\int_{\tau}^{t}\left\langle\boldsymbol{g}(r), \mathbf{u}^{m}(r)\right\rangle d r-C t, \\
& J(t)=\frac{1}{2} E(t)-\int_{\tau}^{t}\langle\boldsymbol{g}(r), \boldsymbol{u}(r)\rangle d r-C t .
\end{aligned}
$$

Obviously, $J_{m}, J$ are non-increasing functions. By (18), we get

$$
J_{m}(t) \rightarrow J(t) \quad \text { a.e. } t \in[\tau, T]
$$

On the one hand, from (21)

$$
E\left(t_{m}\right) \rightarrow E(t) \quad \text { weakly in } \mathbb{Y}
$$

and we get

$$
E\left(t_{0}\right) \leq \lim _{m \rightarrow+\infty} \inf E\left(t_{m}\right)
$$

On the other hand, if $t_{0}=\tau$ we get from Step 3 and (22) with $s=\tau$

$$
\lim _{m \rightarrow+\infty} \sup E\left(t_{m}\right) \leq E\left(t_{0}\right)
$$

so we assume that $t_{0} \geq \tau$. This is important, since we will approach this value $t_{0}$ from the left by a sequence $\left\{\tilde{t}_{k}\right\}$, i.e., $\lim _{k \rightarrow+\infty} \tilde{t}_{k}=t_{0}$ with $\left\{\tilde{t}_{k}\right\}$ being values where (24) holds. Since $E(t)$ is continuous at $t_{0}$, for any $\epsilon$ there is $k_{\epsilon}$ such that

$$
\left|J\left(\tilde{t}_{k}\right)-J\left(t_{0}\right)\right| \leq \epsilon / 2 \quad \forall k \geq k_{\epsilon} .
$$

Then taking $t_{m} \geq t_{\tilde{k}_{\epsilon}}$, as $J_{m}$ is non-increasing and for all $\tilde{t}_{k}$ the convergence (24) holds, and one has

$$
J_{m}\left(t_{m}\right)-J\left(t_{0}\right) \leq\left|J_{m}\left(\tilde{t}_{k_{\epsilon}}\right)-J\left(\tilde{t}_{k_{\epsilon}}\right)\right|+\left|J\left(\tilde{t}_{k_{\epsilon}}\right)-J\left(t_{0}\right)\right|
$$

and with

$$
\int_{\tau}^{t_{m}}\left\langle\boldsymbol{g}(r), \boldsymbol{u}^{m}(r)\right\rangle d r \rightarrow \int_{\tau}^{t_{0}}\langle\boldsymbol{g}(r), \boldsymbol{u}(r)\rangle d r
$$

we get

$$
\lim _{m \rightarrow+\infty} \sup E\left(t_{m}\right) \leq E\left(t_{0}\right)
$$

and

$$
E\left(t_{m}\right) \rightarrow E\left(t_{0}\right) \quad \text { in } \mathbb{Y}
$$


and

$$
\begin{aligned}
& \left(\boldsymbol{u}^{m}, \phi^{m}\right)\left(t_{m}\right) \rightarrow(\boldsymbol{u}, \phi)\left(t_{0}\right) \quad \text { in } \mathbb{Y}, \\
& \left(\boldsymbol{u}^{m}, \phi^{m}\right) \rightarrow(\boldsymbol{u}, \phi) \quad C([\tau, T], \mathbb{Y}) .
\end{aligned}
$$

Thus, we can finally pass to the limit in (11).

The uniqueness of the solution can be obtained in the following way. Consider two weak solutions, $\left(\boldsymbol{u}_{1}, \phi_{1}\right),\left(\boldsymbol{u}_{2}, \phi_{2}\right)$ of (1) with the same initial data, and denote $\boldsymbol{\omega}=\boldsymbol{u}_{1}-\boldsymbol{u}_{2}, \psi=$ $\phi_{1}-\phi_{2},\left(\boldsymbol{u}_{1}, \phi_{1}\right)_{t}=\left(\boldsymbol{u}_{1}, \phi_{1}\right)(t+s),\left(\boldsymbol{u}_{2}, \phi_{2}\right)_{t}=\left(\boldsymbol{u}_{2}, \phi_{2}\right)(t+s)$. We derive as Lemma 3.3 in [5] that

$$
\frac{d y}{d t} \leq \Upsilon(t) y(t)+c\left|G\left(t,\left(\boldsymbol{u}_{1}, \phi_{1}\right)_{t}\right)-G\left(t,\left(\boldsymbol{u}_{2}, \phi_{2}\right)_{t}\right)\right|_{L^{2}}^{2} \leq \Upsilon(t) y(t)+c L_{g}^{2}\left\|(\boldsymbol{\omega}, \psi)_{t}\right\|_{\delta^{\prime}}^{2}
$$

where $c=c_{\Omega}$ is a constant that depends only on $\Omega$ and

$$
\begin{aligned}
y(t)= & |(\boldsymbol{\omega}, \psi)|_{\mathbb{Y}}^{2}, \\
\Upsilon(t)= & c\left(\left\|\boldsymbol{u}_{1}\right\|^{2}+\left(1+\left\|\phi_{1}\right\|^{2}\right)\left|A_{\gamma} \phi_{1}\right|_{L^{2}}^{2}+\left|\boldsymbol{u}_{2}\right|_{L^{2}}^{2}\left\|\boldsymbol{u}_{2}\right\|^{2}\right) \\
& +Q_{1}\left(\left|\phi_{1}\right|_{H^{1}},\left|\phi_{2}\right|_{H^{1}}\right) .
\end{aligned}
$$

As $(\omega, \psi)(\theta)=0$, if $\theta \leq \tau$

$$
\begin{aligned}
\left\|(\omega, \psi)_{t}\right\|_{\delta}^{2} & =\sup _{\theta \leq 0} e^{2 \delta \theta}|(\omega, \psi)(t+\theta)|_{\mathbb{Y}}^{2} \\
& \leq \sup _{\theta \in[\tau-t, 0]}|(\omega, \psi)(t+\theta)|_{\mathbb{Y}}^{2} \\
& \leq \sup _{r \in[\tau, t]}|(\omega, \psi)(r)|_{\mathbb{Y}}^{2}, \quad \forall \tau \leq t \leq T,
\end{aligned}
$$

so

$$
\frac{d y}{d t} \leq \Upsilon(t) y(t)+c L_{g}^{2} \sup _{r \in[\tau, t]} y(r)
$$

we have

$$
y(t) \leq y(0)+\int_{\tau}^{t}\left(\Upsilon(s)+c L_{g}^{2}\right) \sup _{r \in[\tau, s]} y(r) d s .
$$

Now we deduce that

$$
\sup _{r \in[\tau, t]} y(r) \leq y(0)+\int_{\tau}^{t}\left(\Upsilon(s)+c L_{g}^{2}\right) \sup _{r \in[\tau, s]} y(r) d s .
$$

By the Gronwall lemma we finish the proof of uniqueness.

Proposition 1 Assume that $\boldsymbol{g} \in L^{2}\left(\tau, T ; V_{1}^{*}\right), G:[\tau, T] \times C_{\delta}(\mathbb{Y}) \rightarrow\left(L^{2}(\Omega)\right)^{2}$ satisfies assumptions (g1)-(g3). Let us denote by $\left(\boldsymbol{u}_{1}, \phi_{1}\right)\left(\cdot ; \tau, \vartheta_{1}\right),\left(\boldsymbol{u}_{2}, \phi_{2}\right)\left(\cdot ; \tau, \vartheta_{2}\right)$ the weak solutions 
corresponding to the initial data $\vartheta_{1}$ and $\vartheta_{2}$. Then the following continuity properties hold:

$$
\begin{aligned}
& \max _{r \in[\tau, t]}\left|\left(\boldsymbol{u}_{1}, \phi_{1}\right)-\left(\boldsymbol{u}_{2}, \phi_{2}\right)\right|_{\mathbb{Y}}^{2} \leq\left(\left|\vartheta_{1}(0)-\vartheta_{2}(0)\right|^{2}+\frac{L_{g}}{2 \delta}\left\|\vartheta_{1}-\vartheta_{2}\right\|_{\delta}^{2}\right) e^{\int_{\tau}^{t}\left(3 L_{g}+\Upsilon(s)\right) d s}, \\
& \left\|\left(\boldsymbol{u}_{1}, \phi_{1}\right)_{t}-\left(\boldsymbol{u}_{2}, \phi_{2}\right)_{t}\right\|_{\delta}^{2} \leq\left(1+\frac{L_{g}}{2 \delta}\left\|\vartheta_{1}-\vartheta_{2}\right\|_{\delta}^{2}\right) e^{\int_{\tau}^{t}\left(3 L_{g}+\Upsilon(s)\right) d s} .
\end{aligned}
$$

Proof Arguing as in the proof of Theorem 1, we have

$$
\frac{1}{2} \frac{d y}{d t} \leq \Upsilon(t) y(t)+\left(G\left(t,\left(\boldsymbol{u}_{1}, \phi_{1}\right)_{t}\right)-G\left(t,\left(\boldsymbol{u}_{2}, \phi_{2}\right)_{t}\right), \omega\right),
$$

where

$$
\begin{aligned}
y(t)= & \left|\left(\boldsymbol{u}_{1}-\boldsymbol{u}_{2}, \phi_{1}-\phi_{2}\right)\right|_{\mathbb{Y}}^{2}=|(\boldsymbol{\omega}, \psi)|_{\mathbb{Y}}^{2}, \\
\Upsilon(t)= & c\left(\left\|\boldsymbol{u}_{1}\right\|^{2}+\left(1+\left\|\phi_{1}\right\|^{2}\right)\left|A_{\gamma} \phi_{1}\right|_{L^{2}}^{2}+\left|\boldsymbol{u}_{2}\right|_{L^{2}}^{2}\left\|\boldsymbol{u}_{2}\right\|^{2}\right) \\
& +Q_{1}\left(\left|\phi_{1}\right|_{H^{1}},\left|\phi_{2}\right|_{H^{1}}\right) .
\end{aligned}
$$

As

$$
\begin{aligned}
\left\|(\omega, \psi)_{t}\right\|_{\delta}^{2}= & \sup _{\theta \leq 0} e^{2 \delta \theta}|(\omega, \psi)(t+\theta)|_{\mathbb{Y}}^{2} \\
= & \max _{\{}\left\{\sup _{\theta \in(-\infty, \tau-t]} e^{2 \delta \theta}\left|\vartheta_{1}(t-\tau+\theta)-\vartheta_{2}(t-\tau+\theta)\right|_{\mathbb{Y}}^{2},\right. \\
& \left.\sup _{\theta \in[\tau-t, 0]} e^{2 \delta \theta}|(\omega, \psi)(t+\theta)|_{\mathbb{Y}}^{2}\right\} \\
& \leq \max \left\{e^{2 \delta(\tau-t)}\left\|\vartheta_{1}-\vartheta_{2}\right\|_{\delta}^{2}, \max _{\theta \in[\tau, t]}|(\omega, \psi)(\theta)|_{\mathbb{Y}}^{2}\right\}
\end{aligned}
$$

we conclude that

$$
\begin{aligned}
\frac{1}{2} y \leq & \frac{1}{2} y(0)+L_{g}\left\|\vartheta_{1}-\vartheta_{2}\right\|_{\delta} \int_{\tau}^{t} e^{\delta(\tau-s)}|\omega(s)| d s \\
& +L_{g} \int_{\tau}^{t}|\omega(s)| \max _{\theta \in[\tau, t]}|(\omega, \psi)(\theta)|_{\mathbb{Y}} d s+\int_{\tau}^{t} \Upsilon(s) y(s) d s .
\end{aligned}
$$

Substituting $t$ by $r \in[\tau, t]$ and considering the maximum when varying this $r$ we can conclude that

$$
\max _{r \in[\tau, t]} y \leq y(0)+\frac{L_{g}}{2 \delta}\left\|\vartheta_{1}-\vartheta_{2}\right\|_{\delta}^{2}+\int_{\tau}^{t}\left(3 L_{g}+\Upsilon(s)\right) \max _{r \in[\tau, s]} y(r) d s
$$

Then by the Gronwall lemma, we get the result:

$$
\max _{r \in[\tau, t]}\left|\left(\mathbf{u}_{1}, \phi_{1}\right)-\left(\mathbf{u}_{2}, \phi_{2}\right)\right|_{\mathbb{Y}}^{2} \leq\left(\left|\vartheta_{1}(0)-\vartheta_{2}(0)\right|^{2}+\frac{L_{g}}{2 \delta}\left\|\vartheta_{1}-\vartheta_{2}\right\|_{\delta}^{2}\right) e^{\int_{\tau}^{t}\left(3 L_{g}+\Upsilon(s)\right) d s} .
$$

With equations (26) and (27), we get the last inequality. 
Proposition 2 Assume that $\boldsymbol{g} \in L^{2}\left(\tau, T ; V_{1}^{*}\right), G:[\tau, T] \times C_{\delta}(\mathbb{Y}) \rightarrow\left(L^{2}(\Omega)\right)^{2}$ satisfies assumptions (g1)-(g3). Let us denote $(\boldsymbol{u}, \phi)(\cdot ; s, \vartheta)$ the solution of $(1)$ with initial time s. Then, for each $t \in[\tau, T]$ and $\vartheta \in C_{\delta}(\mathbb{Y})$ fixed, the mapping $s \rightarrow(\boldsymbol{u}, \phi) t(\cdot ; s, \vartheta) \in C_{\delta}(\mathbb{Y}), s \in[t, \tau]$ is continuous.

The method of proof is similar to Proposition 7 in [9].

\section{Existence of pullback attractors}

In this section we will prove the existence of a pullback attractor for the problem (1) with the distributed delay under additional assumptions. We firstly recall some basic definitions and main results that we will use later about properties required of a process for a non-autonomous dynamical system in order to have a pullback attractor. These results can be found in [14] and [15] and here we only reproduce the statements for the sake of completeness.

Definition 2 Let $X$ be a complete metric space. A family of mappings $\{U(t, \tau), t, \tau \in R, t \geq$ $\tau\} \subset C^{0}(X, X)$ is said to be a process in $X$ if $U(t, \tau) U(\tau, r)=U(t, r)$ for any $\tau \leq r \leq t$, and $U(\tau, \tau)=I d$ for all $\tau$. The process $U(\cdot, \cdot)$ is said to be continuous if the mapping $(t, \tau) \rightarrow$ $U(t, \tau) x$ is continuous for all $x \in X$.

Corollary 1 Assume that $g \in L_{\mathrm{loc}}^{2}\left(\mathbb{R} ; V_{1}^{*}\right), G: \mathbb{R} \times C_{\delta}(\mathbb{Y}) \rightarrow\left(L^{2}(\Omega)\right)^{2}$ satisfies assumptions (g1)-(g3) for any $\tau<T$. Then the bi-parametric family of mappings $U(t, \tau): C_{\delta}(\mathbb{Y}) \rightarrow C_{\delta}(\mathbb{Y})$ with $t \geq \tau$, defined by

$$
U(t, \tau) \vartheta=(\boldsymbol{u}, \phi)_{t},
$$

where $(\boldsymbol{u}, \phi)(\cdot ; \tau, \vartheta)$ is the unique weak solution of $(1)$, defines a semi-process on $C_{\delta}(\mathbb{Y})$.

Proof The proof is a consequence of Theorem 1 and Proposition 1.

The following result can be obtained analogously to [7], Propositions 2.2, 2.3 with the natural changes in the delay norms.

Proposition 3 Assume that $g \in L_{\mathrm{loc}}^{2}\left(\mathbb{R} ; V_{1}^{*}\right), G: \mathbb{R} \times C_{\delta}(\mathbb{Y}) \rightarrow\left(L^{2}(\Omega)\right)^{2}$ satisfies assumptions (g1)-(g3) for any $\tau<T$. Then for any bounded set $B \subset C_{\delta}(\mathbb{Y})$ :

(1) The set weak of weak solutions $\{(\boldsymbol{u}, \phi)(\cdot ; \tau, \vartheta): \vartheta \in B\}$ is bounded in $L^{\infty}(\tau+\epsilon, T ; V)$ for any $\epsilon>0$ and any $T>\tau+\epsilon$.

(2) Moreover, if $\{\vartheta(0): \vartheta \in B\}$ is bounded in $V$, then $\{(\boldsymbol{u}, \phi)(\cdot ; \tau, \vartheta): \vartheta \in B\}$ is bounded in $L^{\infty}(\tau, T ; \mathbb{Y})$ for all $T>\tau$.

Definition 3 A process $U$ on $X$ is said to be closed if for any $\tau \leq t$, and any sequence $\left\{x_{n}\right\} \subset X$ with $x_{n} \rightarrow x \in X$ and $U(t, \tau) x_{n} \rightarrow y \in X$, then $U(t, \tau) x=y$.

Let us denote by $P(X)$ the family of all nonempty subsets of $X$ and consider a family of nonempty sets $\hat{D_{0}}=\left\{D_{0}(t): t \in R\right\} \subset P(X)$.

Definition 4 We say that a process $U$ on $X$ is pullback $\hat{D}_{0}$-asymptotically compact if for any $t \in R$ and any sequences $\left\{\tau_{n}\right\} \subset(-\infty, t]$ and $\left\{x_{n}\right\} \subset X$ satisfying $\tau_{n} \rightarrow-\infty$ and $x_{n} \in$ $D_{0}\left(\tau_{n}\right)$ for all $n$, the sequence $\left\{U\left(t, \tau_{n}\right) x_{n}\right\}$ is relatively compact in $X$. 
Denote

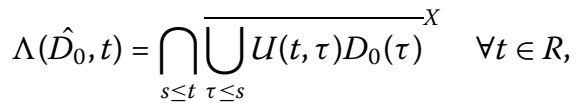

where $\overline{\{\cdots\}}^{X}$ is the closure in $X$.

Given $A, B \subset X$ we denote by $\operatorname{dist}(A, B)$ the Hausdorff semi-distance in $X$ between them, defined as

$$
\operatorname{dist}(A, B)=\sup _{a \in A} \inf _{b \in B} d(a, b)
$$

Let $D$ be a nonempty class of families parameterized in time $\hat{D}=\{D(t): t \in R\} \subset P(X)$. The class $D$ will be called a universe in $P(X)$.

Definition 5 A process $U$ on $X$ is said to be pullback $D$-asymptotically compact if it is pullback $\hat{D}$-asymptotically compact for any $\hat{D} \in D$.

It is said that $\hat{D_{0}}=\left\{D_{0}(t): t \in R\right\} \subset P(X)$ is pullback $D$-absorbing for process $U$ on $X$ if for any $t \in R$ and any $\hat{D} \in D$, there exists a $\tau_{0}(\hat{D}, t) \leq t$ such that

$$
U(t, \tau) D(\tau) \subset D_{0}(t) \quad \forall \tau \leq \tau_{0}(\hat{D}, t)
$$

With the above definitions, we have the main result which is given in [16].

Theorem 2 Consider a closed process $U$, a universe $D$ in $P(X)$, and a family $\hat{D_{0}}=\left\{D_{0}(t)\right.$ : $t \in R\} \subset P(X)$ which is pullback D-absorbing for $U$ and assume that $U$ is pullback $\hat{D_{0}}$ asymptotically compact. Then the family $A(t)$ defined by $A(t)={\overline{\bigcup_{\hat{D} \in D} \Lambda(\hat{D}, t)}}^{X}$ has the following properties:

(1) For any $t \in R$ the set $A(t)$ is a nonempty compact subset of $X$ and $A(t) \subset \Lambda\left(\hat{D_{0}}, t\right)$.

(2) $A$ is pullback $D$-attracting, i.e. $\lim _{r \rightarrow-\infty} \operatorname{dist}(U(t, \tau) D(\tau), A(t))=0$ for all $\hat{D} \in D$ and any $t \in R$.

(3) $A$ is invariant, i.e. $U(t, \tau) A(\tau)=A(t)$ for all $(t, \tau) \in R^{2}$.

(4) If $\hat{D_{0}} \in D$ then $A(t)=\Lambda\left(\hat{D_{0}}, t\right) \subset \overline{D_{0}(t)}$ for all $t \in R$.

The family $A(t)$ is minimal in the sense that if $\hat{C}=\{C(t): t \in R\} \subset P(X)$ is a family of closed sets such that, for any $\hat{D}=\{D(t): t \in R\}, \lim _{r \rightarrow-\infty} \operatorname{dist}(U(t, \tau) D(\tau), C(t))=0$, then $A(t) \subset C(t)$.

Remark 4 Under the assumptions of Theorem 2, the family $A(t)$ is called the minimal pullback $D$-attractor for the process $U$.

Proposition 4 Let $X$ be a connected metric space. Assume that the semi-process $U$ satisfies additionally the requirement that for every $t$ and $x \in X$ the map $\tau \rightarrow U(t, \tau) x, \tau \in(-\infty, t]$ is continuous. If $U$ possesses a pullback attractor $A$, then $A(t)$ is connected for every $t \in R$. 
Theorem 3 Assume that $\mathbf{g} \in L_{\text {loc }}^{2}\left(\mathbb{R} ; V_{1}^{*}\right), G: \mathbb{R} \times C_{\delta}(\mathbb{Y}) \rightarrow\left(L^{2}(\Omega)\right)^{2}$ satisfies (g1)-(g3) for any $\tau<T,(\boldsymbol{u}, \phi)(\cdot ; \tau, \vartheta)$ is the unique weak solution to $(1)$, then the following estimates hold for all $t>\tau$, and any $\sigma \in\left(0, \alpha_{1}\right)$ such that $\left(\alpha_{1}-\sigma\right) \lambda_{1} \leq \delta$ :

$$
\begin{aligned}
\|(\boldsymbol{u}, \phi) t\|_{\delta}^{2} \leq & e^{-\left(\left(\alpha_{1}-\sigma\right) \lambda_{1}-L_{g}\right)(t-\tau)}\|E(\vartheta)\|_{\delta} \\
& \quad \int_{\tau}^{t} e^{-\left(\left(\alpha_{1}-\sigma\right) \lambda_{1}-L_{g}\right)(t-s)}\left(\sigma^{-1}\|\boldsymbol{g}(s)\|_{*}^{2}+c_{0}\right) d s \\
\sigma \int_{\tau}^{t}\|(\boldsymbol{u}, \phi)\|^{2} d s \leq & e^{L_{g}(t-\tau)}\|E(\vartheta)\|_{\delta} \\
& +e^{L_{g} t-\left(\alpha_{1}-\sigma\right) \lambda_{1} \tau} \int_{\tau}^{t} e^{-\left(\left(\alpha_{1}-\sigma\right) \lambda_{1}-L_{g}\right) s}\left(\sigma^{-1}\|\boldsymbol{g}(s)\|_{*}^{2}+c_{0}\right) d s
\end{aligned}
$$

where $c_{0}$ is a positive constant.

Proof Multiplying $(10)_{1}$ with $\boldsymbol{u},(10)_{2}$ with $\mu,(10)_{3}$ with $2 \phi$ and adding the resulting equations, we derive as the proof of Theorem 1 that

$$
\frac{d E}{d t}+2 v\|\boldsymbol{u}\|^{2}+2|\mu|_{L^{2}}^{2}=2\left\langle\boldsymbol{u}, \boldsymbol{g}(t)+G\left(t,(\boldsymbol{u}, \phi)_{t}\right)\right\rangle
$$

where

$$
E(t)=|(\boldsymbol{u}, \phi)(t)|_{\mathbb{Y}}^{2}+2\left\langle F_{\gamma}(\phi(t)), 1\right\rangle_{L^{2}}+\alpha_{0}
$$

and $\alpha_{0}$ is a constant large enough to ensure the $E(t)$ is nonnegative.

We can conclude that

$$
\frac{d E}{d t}+2 v\|\boldsymbol{u}\|^{2}+2|\mu|_{L^{2}}^{2} \leq \sigma^{-1}\|\boldsymbol{g}\|_{*}^{2}+\sigma\|\boldsymbol{u}\|^{2}+2 L_{g}\left\|(\boldsymbol{u}, \phi)_{t}\right\|_{\delta}^{2} .
$$

Thus,

$$
\frac{d E}{d t}+(2 v-\sigma)\|\boldsymbol{u}\|^{2}+2|\mu|_{L^{2}}^{2} \leq \sigma^{-1}\|\boldsymbol{g}\|_{*}+2 L_{g}\left\|(\boldsymbol{u}, \phi)_{t}\right\|_{\delta}^{2} .
$$

So

$$
\frac{d E}{d t}+(2 v-\sigma) \lambda_{1}|\boldsymbol{u}|_{L^{2}}^{2}+2|\mu|_{L^{2}}^{2} \leq \sigma^{-1}\|\boldsymbol{g}\|_{*}+2 L_{g}\left\|(\boldsymbol{u}, \phi)_{t}\right\|_{\delta}^{2} .
$$

Set $\alpha_{1}=2 v$ and as we can choose $\sigma>0$, such that $\alpha_{1}>\sigma$ and $c_{0}$ is a positive constant, then

$$
\frac{d E}{d t} \leq-\left(\alpha_{1}-\sigma\right) \lambda_{1} E+\sigma^{-1}\|\boldsymbol{g}\|_{*}+2 L_{g}\left\|(\boldsymbol{u}, \phi)_{t}\right\|_{\delta}^{2}+c_{0}
$$

So

$$
\begin{aligned}
E(t) \leq & e^{-\left(\alpha_{1}-\sigma\right) \lambda_{1}(t-\tau)} E(\tau) \\
& +\int_{\tau}^{t} e^{-\left(\alpha_{1}-\sigma\right) \lambda_{1}(s-\tau)}\left(\sigma^{-1}\|\boldsymbol{g}\|_{*}+2 L_{g}\left\|(\boldsymbol{u}, \phi)_{s}\right\|_{\delta}^{2}+c_{0}\right) d s .
\end{aligned}
$$


Consequently,

$$
\begin{aligned}
\left\|E_{t}\right\|_{\delta} \leq & \max \left\{\sup _{\theta \in(-\infty, \tau-t]} e^{2 \delta \theta} E(\vartheta(t+\theta-\tau)), \sup _{\theta \in[\tau-t, 0]}\left(e^{2 \delta \theta-\left(\alpha_{1}-\sigma\right) \lambda_{1}(t+\theta-\tau)} E(\tau)\right.\right. \\
& \left.\left.+e^{2 \delta \theta} \int_{\tau}^{t+\theta} e^{-\left(\alpha_{1}-\sigma\right) \lambda_{1}(s+\theta-\tau)}\left(\sigma^{-1}\|\boldsymbol{g}(s)\|_{*}^{2}+2 L_{g}\left\|E_{s}\right\|_{\delta}+c_{0}\right) d s\right)\right\}
\end{aligned}
$$

We assume that, moreover, $\sigma$ satisfies $\left(\alpha_{1}-\sigma\right) \lambda_{1} \leq 2 \delta$.

On the one hand

$$
\begin{aligned}
\sup _{\theta \in(-\infty, \tau-t]} e^{2 \delta \theta} E(\vartheta(t+\theta-\tau)) & =\sup _{\theta \leq 0} e^{2 \delta(\theta-(t-\tau))} E(\vartheta(\theta)) \\
& =e^{-2 \delta(t-\tau)}\|E(\vartheta)\|_{\delta} \leq e^{-\left(\alpha_{1}-\sigma\right) \lambda_{1}(t-\tau)}\|E(\vartheta)\|_{\delta}
\end{aligned}
$$

and on the other hand

$$
\sup _{\theta \in[\tau-t, 0]} e^{2 \delta \theta-\left(\alpha_{1}-\sigma\right) \lambda_{1}(t+\theta-\tau)} E(\tau) \leq e^{-\left(\alpha_{1}-\sigma\right) \lambda_{1}(t-\tau)} E(\tau)
$$

and

$$
\begin{aligned}
& \sup _{\theta \in[\tau-t, 0]} e^{2 \delta \theta} \int_{\tau}^{t+\theta} e^{-\left(\alpha_{1}-\sigma\right) \lambda_{1}(s+\theta-\tau)}\left(\sigma^{-1}\|\boldsymbol{g}(s)\|_{*}^{2}+2 L_{g}\left\|E_{s}\right\|_{\delta}+c_{0}\right) d s \\
& \leq \int_{\tau}^{t} e^{-\left(\alpha_{1}-\sigma\right) \lambda_{1}(s-\tau)}\left(\sigma^{-1}\|\boldsymbol{g}(s)\|_{*}^{2}+2 L_{g}\left\|E_{s}\right\|_{\delta}+c_{0}\right) d s
\end{aligned}
$$

so we get

$$
\begin{aligned}
\left\|E_{t}\right\|_{\delta} \leq & e^{-\left(\alpha_{1}-\sigma\right) \lambda_{1}(t-\tau)}\|E(\vartheta)\|_{\delta} \\
& +\int_{\tau}^{t} e^{-\left(\alpha_{1}-\sigma\right) \lambda_{1}(t-\tau)}\left(\sigma^{-1}\|\boldsymbol{g}(s)\|_{*}^{2}+2 L_{g}\left\|E_{s}\right\|_{\delta}+c_{0}\right) d s \quad \forall t \geq \tau .
\end{aligned}
$$

Then by Gronwall's lemma, we have

$$
\begin{aligned}
\left\|(\boldsymbol{u}, \phi)_{t}\right\|_{\delta}^{2} \leq & \left\|E_{t}\right\|_{\delta} \\
\leq & e^{-\left(\left(\alpha_{1}-\sigma\right) \lambda_{1}-L_{g}\right)(t-\tau)}\|E(\vartheta)\|_{\delta} \\
& +\int_{\tau}^{t} e^{-\left(\left(\alpha_{1}-\sigma\right) \lambda_{1}-L_{g}\right)(t-s)}\left(\sigma^{-1}\|\boldsymbol{g}(s)\|_{*}^{2}+c_{0}\right) d s \quad \forall t \geq \tau .
\end{aligned}
$$

With (32) and the above equality we get the result (28).

From now on, we will assume that there exist $0 \leq \sigma \leq \alpha_{1}$ such that $L_{g} \leq\left(\alpha_{1}-\sigma\right) \lambda_{1} \leq \delta$ and

$$
\int_{-\infty}^{0} e^{\beta s}\left(\sigma^{-1}\|\boldsymbol{g}(s)\|_{*}^{2}+c_{0}\right) d s<+\infty
$$


where

$$
\beta=\left(\left(\alpha_{1}-\sigma\right) \lambda_{1}-L_{g}\right)
$$

Remark 5 If we assume that $\boldsymbol{g} \in L_{\text {loc }}^{2}\left(\mathbb{R} ; V_{1}^{*}\right)$, then equation (33) is equivalent to

$$
\int_{-\infty}^{t} e^{-\beta(t-s)}\left(\sigma^{-1}\|\boldsymbol{g}(s)\|_{*}^{2}+c_{0}\right) d s<+\infty, \quad \forall t \in R
$$

Corollary 2 Assume that $\boldsymbol{g} \in L_{\text {loc }}^{2}\left(\mathbb{R} ; V_{1}^{*}\right), G: \mathbb{R} \times C_{\delta}(\mathbb{Y}) \rightarrow\left(L^{2}(\Omega)\right)^{2}$ satisfies assumptions (g1)-(g3) for any $\tau<T$ and condition (33) is satisfied, then the family $\hat{D_{0}}=\left\{D_{0}(t): t \in R\right\}$, with $D_{0}(t)=D_{C_{\delta}(\mathbb{Y})}(0, \rho(t))$, where

$$
\rho^{2}(t)=1+\int_{-\infty}^{t} e^{-\beta(t-s)}\left(\sigma^{-1}\|\boldsymbol{g}(s)\|_{*}^{2}+c_{0}\right) d s
$$

is pullback absorbing for bounded sets for the semi-process $U$.

Proof The proof follows immediately from Theorem 3.

Proposition 5 Under the assumptions of Corollary 2, the semi-process $U$ is $\hat{D}_{0}$ asymptotically compact.

Proof Let $\left(\boldsymbol{u}^{m}, \phi^{m}\right)$ be a sequence in Theorem 1, we will prove this sequence is relatively compact in $C_{\delta}(\mathbb{Y})$.

Let $\tau_{1}(\hat{D}, t, h)<t-h-2$ be such that

$$
e^{-\beta(t-h-2)} e^{\beta \tau}\|E(\vartheta)\|_{\delta} \leq 1 \quad \forall \tau \leq \tau_{1}(\hat{D}, t, h), \vartheta \in D(\tau) .
$$

Consider fixed $\tau \leq \tau_{1}(\hat{D}, t, h)$ and $\vartheta \in D(\tau)$.

Firstly, from the result (28) of Theorem 3 and using the definition of the norm $\|\cdot\|_{\delta}$, we can deduce that

$$
\begin{aligned}
\left|(\boldsymbol{u}, \phi)_{t}\right|_{Y}^{2} & \leq 1+e^{-\beta(t-h-2)} \int_{-\infty}^{t} e^{\beta s}\left(\left(\sigma \lambda_{1}\right)^{-1}|\boldsymbol{g}(s)|^{2}+c_{0}\right) d s \\
& \triangleq \rho_{1}(t) \quad \forall r \in[t-h-2, t] .
\end{aligned}
$$

Secondly, we derive from Theorem 3 by integrating (31) between $r-1$ and $r$

$$
\begin{gathered}
E^{m}(r)-E^{m}(r-1)+\left(\alpha_{1}-\sigma\right) \int_{r-1}^{r}\left\|\boldsymbol{u}^{m}\right\|^{2}+2 \int_{r-1}^{r}\left|\mu^{m}(t)\right|_{L^{2}}^{2} d t \\
\leq \sigma^{-1}\|\boldsymbol{g}\|_{*}^{2}+2 L_{g} \int_{r-1}^{r}\left\|\left(\boldsymbol{u}^{m}, \phi^{m}\right)_{s}\right\|_{\delta}^{2} d s,
\end{gathered}
$$

then from (28) and

$$
|(\boldsymbol{u}(t), \phi(t))|_{\mathbb{Y}}^{2} \leq E(t) \leq Q_{1}\left(|(\boldsymbol{u}(t), \phi(t))|_{\mathbb{Y}}^{2}\right),
$$


where $Q_{1}$ is a monotone non-decreasing function independent of the time and the initial data, we can deduce that

$$
\begin{aligned}
& \left(\alpha_{1}-\sigma\right) \int_{r-1}^{r}\left\|\boldsymbol{u}^{m}\right\|^{2}+2 \int_{r-1}^{r}\left|\mu^{m}(t)\right|_{L^{2}}^{2} d t \\
& \leq \sigma^{-1}\|\boldsymbol{g}\|_{*}^{2}+2 L_{g} \int_{r-1}^{r}\left\|\left(\boldsymbol{u}^{m}, \phi^{m}\right)_{s}\right\|_{\delta}^{2} d s+E^{m}(r-1) \\
& \leq \sigma^{-1}\|\boldsymbol{g}\|_{*}^{2}+2 L_{g} \int_{r-1}^{r}\left(e^{-\beta(s-\tau)}\|E(\vartheta)\|_{\delta}+\int_{\tau}^{s} e^{-\beta(s-\xi)}\left(\left(\sigma \lambda_{1}\right)^{-1}|\boldsymbol{g}(\xi)|^{2}\right) d \xi+c_{0}\right) d s \\
& \quad+Q_{1}\left(\left|\mathbf{u}^{m}(t), \phi^{m}(t)\right|_{Y}^{2}\right) .
\end{aligned}
$$

As $\alpha_{1}-\sigma>0$,

$$
\int_{r-1}^{r}\left(\left\|\boldsymbol{u}^{m}\right\|^{2}+\left|\mu^{m}\right|_{L^{2}}^{2}\right) d t \leq C_{1}
$$

From $\mu=\epsilon A_{\gamma} \phi+\alpha f_{\gamma} \phi$ we have

$$
\epsilon^{2} \int_{r-1}^{r}\left|A_{\gamma} \phi^{m}\right|_{L^{2}}^{2} d t \leq c \int_{r-1}^{r}\left(\left|\mu^{m}\right|_{L^{2}}^{2}+\alpha\left|f_{\gamma} \phi^{m}\right|_{L^{2}}^{2}\right) d t \leq C_{2}
$$

so

$$
\int_{r-1}^{r}\left(\left\|\boldsymbol{u}^{m}\right\|^{2}+\left|A_{\gamma} \phi^{m}\right|_{L^{2}}^{2}\right) d t \leq C_{3}
$$

where $C_{1}, C_{2}$ and $C_{3}$ are constants.

For the rest of the estimates we take the inner product in $H_{1}$ of $(11)_{1}$ with $2 \boldsymbol{A}_{0} \boldsymbol{u}$, the inner product in $L^{2}(M)$ of $(11)_{2}$ with $2 A_{\gamma}^{2} \phi$ and add the resulting equalities; we get (see [12] for the details) the inner product by integrating between $r-1$ and $r$,

$$
\frac{d Y}{d t}+v\left|\boldsymbol{A}_{0} \boldsymbol{u}^{m}\right|_{L^{2}}^{2}+\epsilon\left|A_{\gamma}^{3 / 2} \phi^{m}\right|_{L^{2}}^{2} \leq \Psi(t) Y(t)+\Pi(t)
$$

where

$$
\begin{aligned}
& Y(t)=\left\|\boldsymbol{u}^{m}(t)\right\|^{2}+\left|A_{\gamma} \phi^{m}(t)\right|_{L^{2}}^{2}, \\
& \Psi(t)=c\left(\left|A_{\gamma} \phi^{m}(t)\right|_{L^{2}}^{2}\left|\phi^{m}(t)\right|_{H^{1}}^{2}+\left|\mathbf{u}^{m}\right|_{L^{2}}^{2}\left\|\boldsymbol{u}^{m}\right\|^{2}\right), \\
& \Upsilon(t)=c\left(\left|f_{\gamma}^{\prime}(\phi) \nabla \phi^{m}\right|_{L^{2}}^{2}+|\boldsymbol{g}|_{L^{2}}^{2}\right), \\
& \Pi(t)=\Upsilon(t)+\left|G\left(t,\left(\boldsymbol{u}^{m}, \phi^{m}\right)_{t}\right)\right|_{L^{2}}^{2} .
\end{aligned}
$$

By integrating between $r-1$ and $r$ and from (28) we can obtain

$$
\begin{aligned}
Y(r) \leq & \left(\int_{r-1}^{r} Y(s) d s+\int_{r-1}^{r} \Upsilon(s) d s+L_{g}^{2} \int_{r-1}^{r}\left\|\left(\boldsymbol{u}^{m}, \phi^{m}\right)_{s}\right\|_{\delta}^{2} d s\right) \times \exp \left(\int_{r-1}^{r} \Psi(s) d s\right) \\
\leq & \left(\int_{r-1}^{r} Y(s) d s+\int_{r-1}^{r} \Upsilon(s) d s+L_{g}^{2} \int_{r-1}^{r}\left(e^{-\beta(s-\tau)}\|E(\vartheta)\|_{\delta}\right.\right. \\
& \left.\left.+\int_{\tau}^{s} e^{-\beta(s-\xi)}\left(\left(\sigma \lambda_{1}\right)^{-1}|\boldsymbol{g}(\xi)|^{2}\right) d \xi+c_{0}\right) d s\right) \times \exp \left(\int_{r-1}^{r} \Psi(s) d s\right) .
\end{aligned}
$$


Recalling (3), (4) and (34), and employing standard Sobolev inequalities, it is easy to check that there exist positive constants $a_{i}, i=1,2,3$, such that

$$
\begin{aligned}
\int_{r-1}^{r} \Psi(s) d s & \equiv a_{1}<\infty ; \quad \int_{r-1}^{r} \Upsilon(s) d s \equiv a_{2}<\infty, \\
\int_{r-1}^{r} Y(s) d s & \equiv a_{3}<\infty .
\end{aligned}
$$

Then we get

$$
Y(r)=\left\|\boldsymbol{u}^{m}(r)\right\|^{2}+\left|A_{\gamma} \phi^{m}(r)\right|_{L^{2}}^{2} \quad \text { is bounded } \forall r \in[t-h-1, t] .
$$

Letting $(\boldsymbol{u}, \phi)=(\cdot ; t-s, \vartheta)$ we get

$$
\left\|\left(\boldsymbol{u}^{m}, \phi^{m}\right)_{t}\right\|_{\mathbb{V}} \quad \text { is bounded } \forall r \in[t-h-1, t] .
$$

Thirdly, we have

$$
v\left|\boldsymbol{A}_{0} \boldsymbol{u}^{m}\right|_{L^{2}}^{2}+\epsilon\left|A_{\gamma}^{3 / 2} \phi^{m}\right|_{L^{2}}^{2} \leq-\frac{d Y}{d t}+\Psi(t) Y(t)+\Pi(t) .
$$

By integrating between $r-1$ and $r$, we obtain

$$
\begin{aligned}
& \int_{r-1}^{r}\left(v\left|\boldsymbol{A}_{0} \boldsymbol{u}^{m}\right|_{L^{2}}^{2}+\epsilon\left|A_{\gamma}^{3 / 2} \phi^{m}\right|_{L^{2}}^{2}\right) d s \\
& \quad \leq Y(r-1)+\int_{r-1}^{r}(\Psi(s) Y(s)+\Pi(s)) d s \\
& \quad \leq Y(r-1)+\int_{r-1}^{r}\left(\Psi(s) Y(s)+\Upsilon(s)+L_{g}^{2}\left\|\left(\mathbf{u}^{m}, \phi^{m}\right)_{s}\right\|_{\delta}^{2}\right) d s .
\end{aligned}
$$

With (28) we have

$$
\int_{r-1}^{r}\left(v\left|\boldsymbol{A}_{0} \mathbf{u}^{m}\right|_{L^{2}}^{2}+\epsilon\left|A_{\gamma}^{3 / 2} \phi^{m}\right|_{L^{2}}^{2}\right) d s \quad \text { is bounded } \forall r \in[t-h, t] .
$$

Similar to the proof of Theorem 1, we also conclude that

$$
\int_{r-1}^{r}\left|\frac{d}{d t}\left(\mathbf{u}^{m}, \phi^{m}\right)\right|^{2} d s \quad \text { is bounded } \forall r \in[t-h, t]
$$

so $\left(\boldsymbol{u}^{m}(t), \phi^{m}(t)\right)$ is bounded in $L^{\infty}(t-h-1, t ; \mathbb{Y}) \cap L^{2}(t-h-1, t ; \mathbb{V})$ with $\frac{d}{d t}\left(\boldsymbol{u}^{m}, \phi^{m}\right)$ is bounded in $L^{2}\left(t-h-1, t ; \mathbb{V}_{1}^{*}\right) \times L^{2}\left(t-h-1, t ; \mathbb{V}_{1}^{*}\right)$ and for a subsequence (relabeled the same) the following convergences hold:

$$
\left\{\begin{array}{l}
\left(\boldsymbol{u}^{m}, \phi^{m}\right) \stackrel{*}{\rightarrow}(\boldsymbol{u}, \phi) \quad \text { weakly star in } L^{\infty}(t-h-1, t ; \mathbb{Y}), \\
\left(\boldsymbol{u}^{m}, \phi^{m}\right) \rightarrow(\boldsymbol{u}, \phi) \quad \text { weakly in } L^{2}(t-h-1, t ; \mathbb{V}), \\
\frac{d}{d t}\left(\boldsymbol{u}^{m}, \phi^{m}\right) \rightarrow \frac{d}{d t}(\boldsymbol{u}, \phi) \quad \text { weakly in } L^{2}\left(t-h-1, t ; V_{1}^{*}\right) \times L^{2}\left(t-h-1, t ; V_{2}^{*}\right), \\
\left(\boldsymbol{u}^{m}, \phi^{m}\right) \rightarrow(\boldsymbol{u}, \phi) \quad \text { strongly in } L^{2}(t-h-1, t ; \mathbb{Y}) \\
\left(\boldsymbol{u}^{m}(t), \phi^{m}(t)\right) \rightarrow(\boldsymbol{u}(t), \phi(t)) \quad \text { a.e., } t \in(t-h-1, t)
\end{array}\right.
$$


Then the following proof can be obtained analogously to Step 4 of Theorem 1 . To avoid unnecessary repetition, we skip the proof.

Theorem 4 Assume that $\boldsymbol{g} \in L_{\text {loc }}^{2}\left(\mathbb{R} ; V_{1}^{*}\right), G: \mathbb{R} \times C_{\delta}(\mathbb{Y}) \rightarrow\left(L^{2}(\Omega)\right)^{2}$ satisfies assumptions (g1)-(g3) for any $\tau<T$. Also, suppose that $L_{g} \leq\left(\alpha_{1}-\sigma\right) \lambda_{1}$. Then the semi-process $U$ defined in $C_{\delta}(\mathbb{Y})$ associated to (10) has a pullback attractor $A=\{A(t)\}$. Moreover, every $A(t)$ is connected in $C_{\delta}(\mathbb{Y})$.

Proof The existence of the pullback attractor is a direct consequence of Theorem 2, Corollary 1, Corollary 2 and Proposition 5. The connectedness follows from Propositions 2 and 4 and the fact that the space $C_{\delta}(\mathbb{Y})$ is connected.

\section{Acknowledgements}

The paper was supported by the Fundamental Research Funds for the Central Universities (2013B07314). The author also wants to thank the anonymous referees for their valuable comments on the paper.

\section{Competing interests}

The author declares that there is no competition of interests regarding the publication of this paper.

\section{Authors' contributions}

The author wrote the paper and approved the final manuscript.

\section{Authors' information}

Min Yang (1978-), female, lecturer, engaged in the research of numerical solution of partial differential equations.

\section{Publisher's Note}

Springer Nature remains neutral with regard to jurisdictional claims in published maps and institutional affiliations.

Received: 16 February 2017 Accepted: 3 August 2017 Published online: 15 August 2017

References

1. Anderson, DM, McFadden, GB, Wheeler, AA: Diffuse-interface methods in fluid mechanics. Annu. Rev. Fluid Mech 30(1), 139-165 (1998)

2. Feng, JJ, Liu, C, Shen, J, Yue, P: An energetic variational formulation with phase field methods for interfacial dynamics of complex fluids. In: Advantages and Challenges, vol. 141, pp. 1-26. Springer, New York (2005)

3. Blesgen, T: A generalization of the Navier-Stokes equation to two-phase flows. J. Appl. Phys. 32(10), 1119-1123 (1999)

4. Feireisl, E, Petzeltova, H, Rocca, E, Schimperna, G: Analysis of a phase-field model for two-phase compressible fluids. Math. Models Methods Appl. Sci. 20(7), 1129-1160 (2010)

5. Gal, CG, Grasselli, M: Longtime behavior for a model of homogeneous incompressible two-phase flows. Discrete Contin. Dyn. Syst. 28(1), 1-39 (2010)

6. Gal, CG, Grasselli, M: Asymptotic behavior of a Cahn-Hilliard-Navier-Stokes system in 2D. Ann. Inst. Henri Poincaré, Anal. Non Linéaire 27(1), 401-436 (2010)

7. Medjo, TT: Pullback attractors for a non-autonomous homogeneous two-phase flow model. J. Differ. Equ. 253(6), 1779-1806 (2012)

8. Caraballo, T, Real, J: Attractors for 2D-Navier-Stokes models with delays. J. Differ. Equ. 205(2), 271-297 (2004)

9. Marín-Rubio, P, Real, J, Valero, J: Pullback attractors for a two-dimensional Navier-Stokes model in an infinte delay case. Nonlinear Anal. 74(5), 2012-2030 (2011)

10. García-Luengo, J, Marín-Rubio, P, Real, J: Regularity of pullback attractors and attraction in $\mathrm{H} 1$ in arbitrarily large finite intervals for 2D Navier-Stokes equations with infinite delay. Discrete Contin. Dyn. Syst. 34(1), 181-201 (2014)

11. Medjo, TT: A two-phase flow model with delays. Discrete Contin. Dyn. Syst. 21, 2263-2285 (2016)

12. Medjo, TT: Attractors for a two-phase flow model with delays. Differ. Integral Equ. 29, 1071-1092 (2016)

13. Temam, R: Infinite dimensional dynamical systems in mechanics and physics. Appl. Math. Sci. 68(5), 2135-2143 (2000)

14. Kloeden, PE, Schmalfuss, B: Nonautonomous systems, cocycle attractors and variable time-step discretization. Numer. Algorithms 14(1), 141-152 (1997)

15. Kloeden, PE, Stonier, DJ: Cocycle attractors in nonautonomously perturbed differential equations. Dyn. Contin. Discrete Impuls. Syst. 4, 211-226 (1998)

16. García-Luengo, J, Marín-Rubio, P, Real, J: Pullback attractors in V for non-autonomous 2D-Navier-Stokes equations and their tempered behaviour. J. Differ. Equ. 252(8), 4333-4356 (2012) 